\title{
Diffusivity Measurements Differentiate Benign from Malignant Lesions in Patients with Peripheral Neuropathy or Plexopathy
}

\author{
E.L. Yuh, S. Jain Palrecha, G.M. Lagemann, M. Kliot, P.R. Weinstein, N.M. Barbaro, and C.T. Chin
}

\begin{abstract}
BACKGROUND AND PURPOSE: Peripheral nerve disorders caused by benign and malignant primary nerve sheath tumors, infiltration or compression of nerves by metastatic disease, and postradiation neuritis demonstrate overlapping features on conventional MR imaging but require vastly different therapeutic approaches. We characterize and compare diffusivities of peripheral nerve lesions in patients undergoing MR neurography for peripheral neuropathy or brachial or lumbosacral plexopathy.
\end{abstract}

MATERIALS AND METHODS: Twenty-three patients, referred for MR neurography at our institution between 2003 and 2009 for a peripheral mononeuropathy or brachial or lumbosacral plexopathy and whose examinations included DWI, received a definitive diagnosis, based on biopsy results or clinical and imaging follow-up, for a masslike or infiltrative peripheral nerve or plexus lesion suspicious for tumor. Mean ADC values were determined within each lesion and compared across 3 groups (benign lesions, malignant lesions, and postradiation changes).

RESULTS: Both ANOVA and Kruskal-Wallis tests demonstrated a statistically significant difference in ADC values across the 3 groups $(P=$ $.000023, P=.00056$, respectively). Post hoc pair-wise comparisons showed that the ADC within malignant tumors differed significantly from that within benign tumors and postradiation changes. ADC within benign tumors and postradiation changes did not differ significantly from each other.

CONCLUSIONS: DWI may be highly effective for the differentiation of benign from malignant peripheral nerve masslike or infiltrative lesions.

$\mathbf{P}$ eripheral neuropathies can be divided into mononeuropathies, polyneuropathies, and plexopathies. Patients present with pain, sensory symptoms, and/or motor deficits in the distribution of a single peripheral nerve, multiple peripheral nerves, or a nerve plexus. Mononeuropathies affect a single peripheral nerve. Polyneuropathies affect multiple peripheral nerves. In plexopathies, symptoms are localized to the brachial or lumbosacral plexus.

Received February 22, 2014; accepted after revision June 23.

From the Departments of Radiology and Biomedical Imaging (E.L.Y., C.T.C.) and Neurological Surgery (P.R.W.), University of California at San Francisco, San Francisco, California; San Leandro Medical Center (S.J.P.), The Permanente Medical Group, San Leandro, California; Department of Radiology (G.M.L.), University of Pittsburgh, Pittsburgh, Pennsylvania; Department of Neurosurgery (M.K.), Northwestern University Feinberg School of Medicine, Chicago, Illinois; and Goodman Campbell Brain and Spine (N.M.B.) and Department of Neurological Surgery (N.M.B.), Indiana University, Indianapolis, Indiana. E.L. Yuh and S. Jain Palrecha contributed equally to this work.

Please address correspondence to Cynthia T. Chin, MD, Department of Radiology and Biomedical Imaging, University of California at San Francisco, 505 Parnassus Ave, Box 0628, San Francisco, CA 94143-0628; e-mail: cynthia.t.chin@ucsf.edu

三 Indicates article with supplemental on-line table.

http://dx.doi.org/10.3174/ajnr.A4080
Polyneuropathies are generally attributable to systemic diseases (eg, diabetes and vitamin deficiencies), while mononeuropathies are most often due to trauma, nerve compression syndromes that occur at a few characteristic anatomic locations, or mass lesions. History and physical examination, supplemented in a subset of cases by laboratory studies, electrodiagnostic studies, and neuroimaging, are the main tools for diagnostic evaluation. In patients with a classic compression mononeuropathy, such as median nerve compression at the carpal tunnel, the diagnosis can often be made clinically and corroborated by needle electromyography, nerve conduction, and/or imaging studies. ${ }^{1-4}$ For mononeuropathies involving nerves not typically susceptible to compression syndromes, imaging can play an essential role in identifying the lesion and guiding management.

Plexopathies give rise to motor and/or sensory deficits in an extremity. Most brachial plexopathies (75\%) are attributable to postradiation changes, primary and metastatic lung cancer, or metastatic breast cancer. ${ }^{5}$ Common causes of lumbosacral plexopathy are primary and metastatic tumor, including cervical, endometrial, ovarian, prostate, testicular, and colorectal cancer; 
postradiation changes; and diabetes. ${ }^{6}$ For patients with a history of radiation for malignancy, recurrent tumor with nerve invasion must be distinguished from radiation plexopathy; both can develop months to years following therapy and can have similar clinical presentations. ${ }^{6}$

Although benign and malignant primary nerve sheath tumors, infiltration of nerves by metastatic disease, and postradiation neuritis require different therapeutic approaches, they also demonstrate overlapping features on MR imaging, including T2 hyperintensity, focal enlargement, and enhancement. ${ }^{7,8}$ Diffusivity measurements from DWI may be helpful in differentiating distinct pathologic entities. In prior studies, DWI was useful in differentiating malignant and benign peripheral nerve sheath tumors, ${ }^{9}$ retroperitoneal masses, ${ }^{10}$ head and neck tumors, ${ }^{11,12}$ and lymph nodes. ${ }^{13,14}$ Other studies have demonstrated differences in the diffusivities of adult ${ }^{15}$ or pediatric brain tumors ${ }^{16}$ that correlate with tumor grade and/or histologic type. In this study, we focus on masslike or infiltrative lesions of the peripheral nerves detected by MR imaging in patients presenting clinically with a peripheral mononeuropathy or plexopathy. We characterize and compare the diffusivities of these lesions and demonstrate significant differences among benign and malignant peripheral nerve tumors and postradiation changes.

\section{MATERIALS AND METHODS Study Population}

Twenty-three patients referred for MR neurography at our institution between 2003 and 2009 by neurologists, neurosurgeons, and oncologists for a clinical indication of a peripheral mononeuropathy or brachial or lumbosacral plexopathy and who received a definitive diagnosis of a masslike or infiltrative nerve lesion based on biopsy results, long-term clinical and imaging follow-up, or intermediate-term follow-up supplemented by PET, neurologic examination, and/or nerve conduction studies were included in the study population. We included patients with noncystic "mass"-like lesions, defined as noncystic lesions with a diameter at least $50 \%$ larger than that of the apparent nerve of origin as well as patients with more infiltrative lesions consisting of more subtle nerve thickening and/or effacement of normally visualized interfascicular and surrounding fat planes without the presence of a well-defined "mass." Study participants were divided into 3 groups according to the final most likely pathologic diagnosis. Group $1(n=10)$ consisted of benign lesions; Group 2 $(n=7)$, malignant tumors; and Group $3(n=6)$, postradiation changes, without evidence of residual tumor.

\section{MR Neurography}

MR imaging sequences performed at 1.5T (Gyroscan Intera 1.5T; Philips Healthcare, Best, the Netherlands) included axial and coronal STIR $(\mathrm{TR}=2200 \mathrm{~ms}, \mathrm{TE}=20 \mathrm{~ms}, \mathrm{TI}=160 \mathrm{~ms}, \mathrm{NEX}=4$, $\mathrm{FOV}=22$, matrix $=256 \times 192$, slice thickness $/$ gap $=3 / 0.3 \mathrm{~mm})$, $\mathrm{T} 1$ and fat-saturated postgadolinium T1-weighted spin-echo $(\mathrm{TR}=500, \mathrm{TE}=14, \mathrm{NEX}=3)$, and DWI (single-shot echoplanar imaging, 6 directions, $\mathrm{TR}=2 \mathrm{~ms} \times$ pulse-pulse interval, $\mathrm{TE}=15 \mathrm{~ms}, \mathrm{FOV}=22$, matrix $=256 \times 144$, slice thickness $/$ gap $=5.0 / 0.5 \mathrm{~mm}$, b-value $=400 \mathrm{~s} / \mathrm{mm}^{2}$ ). ADC maps were cal-

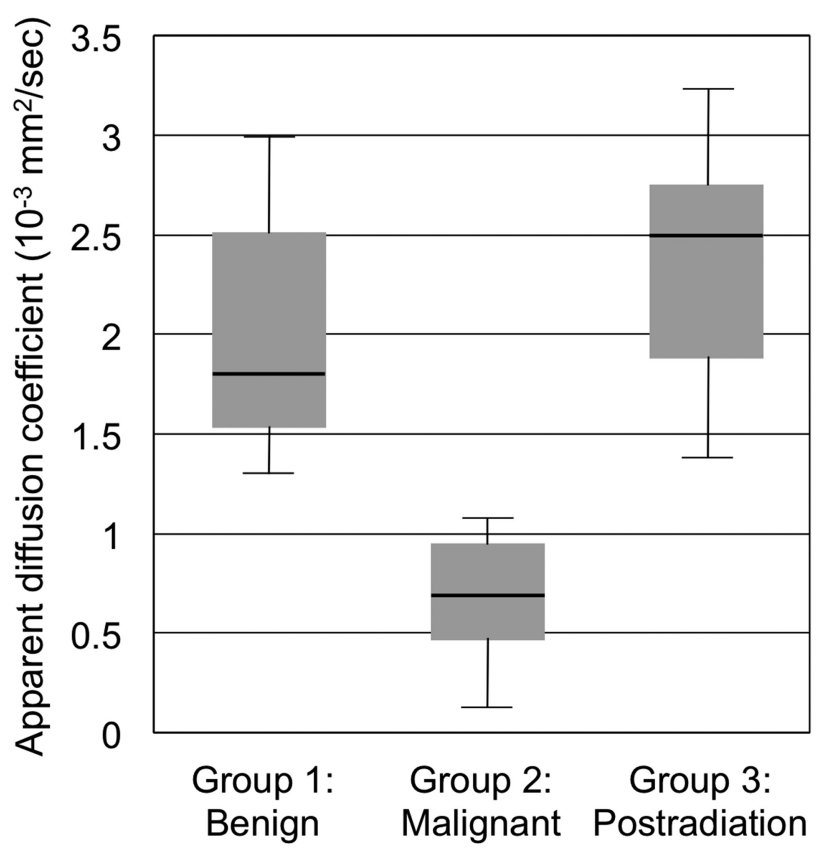

FIG 1. Boxplot shows median (thick horizontal lines within boxes), interquartile range (heights of boxes), and smallest and largest (lines protruding from boxes) ADC values within Group 1 (benign masses), Group 2 (malignant lesions), and Group 3 (postradiation changes). Group 1 ( $n=10$ ) included schwannomas and neurofibromas; Group $2(n=7)$ included 1 rhabdomyosarcoma, 1 malignant peripheral nerve sheath tumor, 2 cases of metastatic breast cancer, 1 case of metastatic renal cell carcinoma, 1 diffuse large B-cell lymphoma, and 1 case of acute lymphoblastic leukemia. Group 3 $(n=6)$ consisted of postradiation changes with no evidence for residual tumor. Group 3 (postradiation changes) demonstrated the highest median $A D C$ value of $2.50 \times 10^{-3} \mathrm{~mm}^{2} / \mathrm{s}$ (interquartile range $=$ $0.87 \times 10^{-3} \mathrm{~mm}^{2} / \mathrm{s}$, minimum $=1.39 \times 10^{-3} \mathrm{~mm}^{2} / \mathrm{s}$, maximum $=$ $3.22 \times 10^{-3} \mathrm{~mm}^{2} / \mathrm{s}$ ), followed closely by benign lesions (Group 1) with a median $A D C$ value of $1.81 \times 10^{-3} \mathrm{~mm}^{2} / \mathrm{s}$ (interquartile range $=0.98 \times 10^{-3} \mathrm{~mm}^{2} / \mathrm{s}$, minimum $=1.30 \times 10^{-3} \mathrm{~mm}^{2} / \mathrm{s}$, maximum $=2.97 \times 10^{-3} \mathrm{~mm}^{2} / \mathrm{s}$ ). Malignant lesions (Group 2) recorded the lowest median ADC value of $0.69 \times 10^{-3} \mathrm{~mm}^{2} / \mathrm{s}$ (interquartile range $=0.49 \times 10^{-3} \mathrm{~mm}^{2} / \mathrm{s}$, minimum $=0.26 \times 10^{-3} \mathrm{~mm}^{2} / \mathrm{s}$, maximum $=1.08 \times 10^{-3} \mathrm{~mm}^{2} / \mathrm{s}$ ).

culated using the Philips vendor software on the MR imaging scanner console immediately after acquisition of DWI data.

Mean and SD of ADC values within approximately 1-cm ROIs drawn within the lesions were determined independently by 2 board-certified radiologists. Each radiologist drew multiple ROIs for lesions that exceeded $3 \mathrm{~cm}$ and averaged the means within these ROIs, to avoid limited regional sampling of spatially heterogeneous lesions. Nonenhancing T2 hyperintense areas suspicious for cystic or necrotic areas were not included within any ROI. Volumes of masslike lesions were approximated by using the volume formula for an ellipsoid; volume $=(4 / 3) \times \pi \times a \times b \times c$, where $\mathrm{a}, \mathrm{b}$, and $\mathrm{c}$ were orthogonal linear dimensions of the lesion measured by one radiologist. (Volume measurements were not performed on infiltrative lesions that consisted of more subtle nerve thickening without a well-defined mass with a diameter at least $50 \%$ larger than that of the nerve of origin.)

\section{Statistical Analysis}

Intrarater reliability for ADC measurements was determined through calculation of the Pearson correlation coefficient be- 

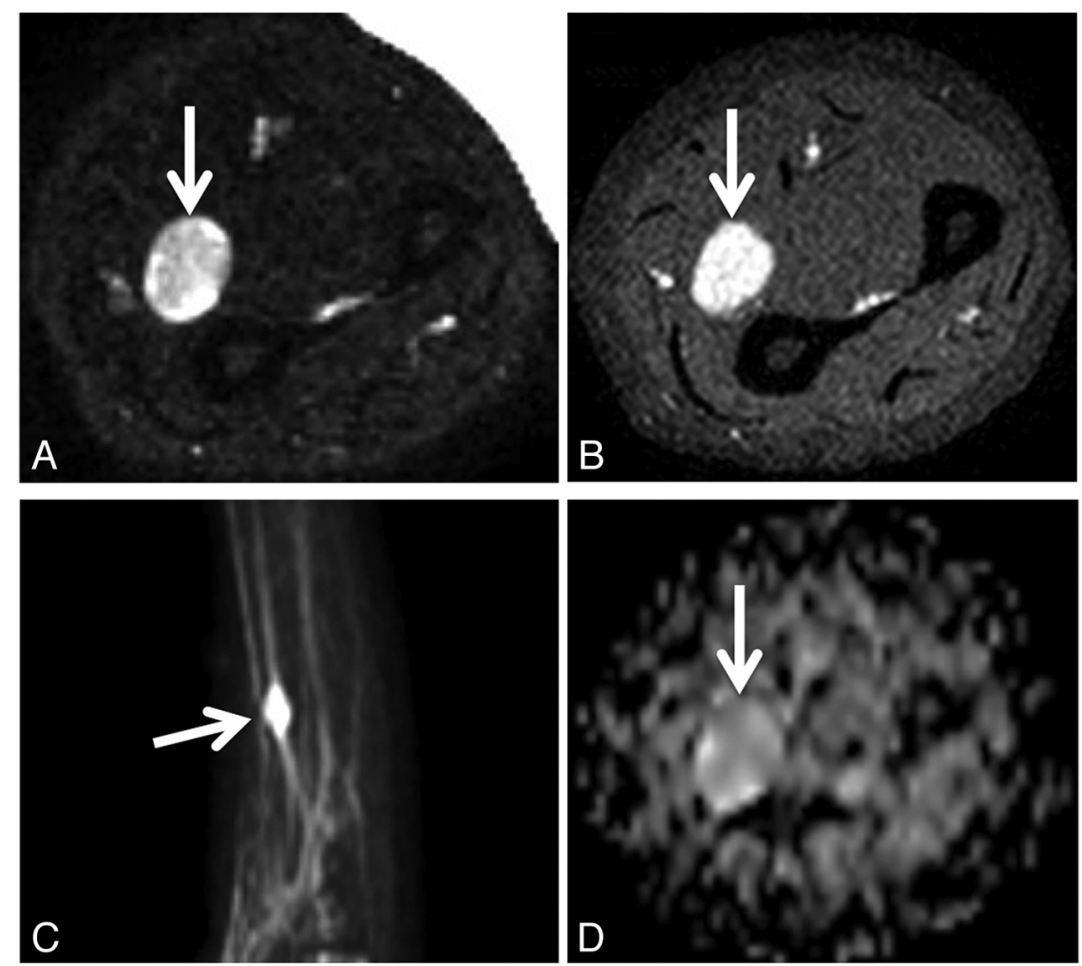

FIG 2. Biopsy-proven schwannoma of the left median nerve in a 48-year-old woman presenting with left upper extremity pain and paresthesias. Axial fat-suppressed T2 (A), axial fat-saturated postgadolinium T1 (B), maximum-intensity-projection DWI (C), and axial ADC (D) demonstrate a vividly enhancing, heterogeneously $T 2$ hyperintense mass (arrows) along the median nerve. The ADC value within the mass was $2.1 \pm 0.36 \times 10^{-3}$ $\mathrm{mm}^{2} / \mathrm{s}$. The lesion was resected due to progressive symptoms.
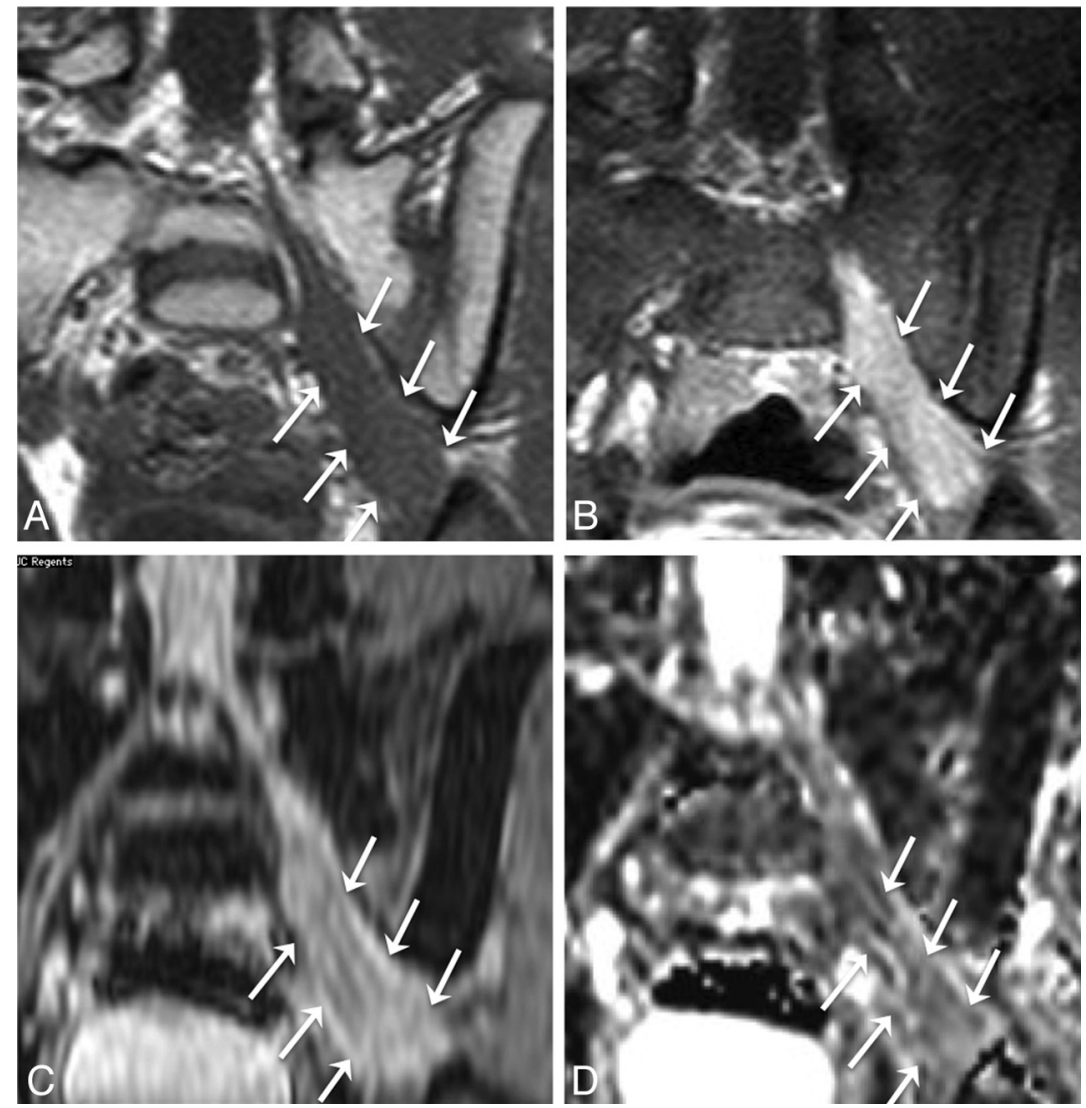

FIG 3. Biopsy-proven neurofibroma of the left S1 and sciatic nerves in a 6-year-old boy with left foot drop. Coronal Tl (A), coronal fat-saturated postgadolinium $T\urcorner(B)$, oblique reformatted coronal STIR (C), and coronal ADC (D) demonstrate enhancing masslike T2 hyperintense soft tissue (arrows) along the left S1 nerve and left sciatic nerve. The lesion was treated with radiation therapy. 

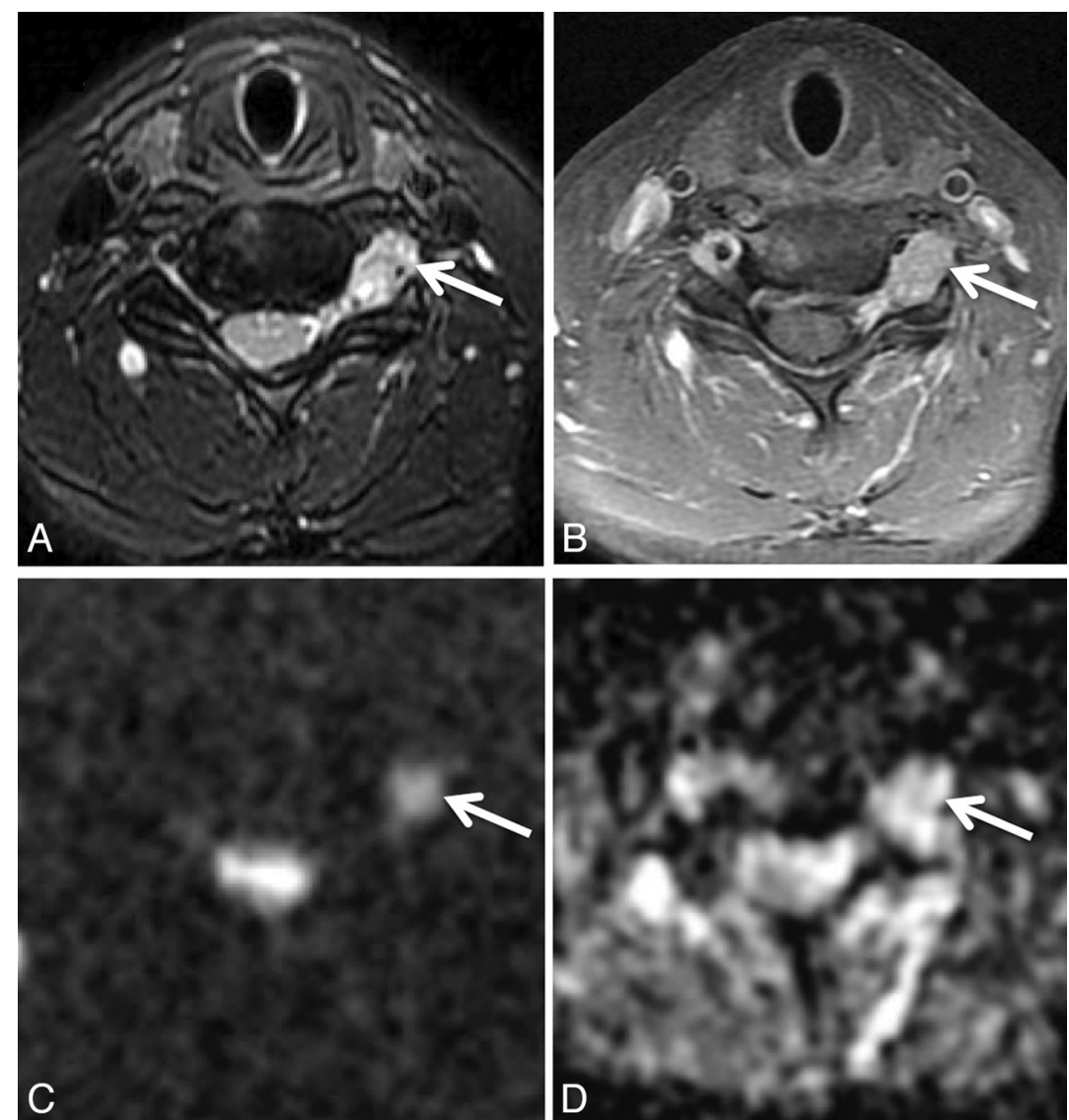

FIG 4. Metastatic renal cell carcinoma in a 46-year-old woman with left arm weakness. Axial STIR ( $A$ ), axial fat-saturated postgadolinium T1 (B), axial DWI (C), and axial ADC (D) demonstrate a T2 hyperintense, enhancing mass (arrows) along the left C6 nerve just outside the left C5-C6 neural foramen. $A D C$ within the mass was $1.08 \pm 0.09 \times 10^{-3} \mathrm{~mm}^{2} / \mathrm{s}$. The mass was subtotally resected and irradiated.
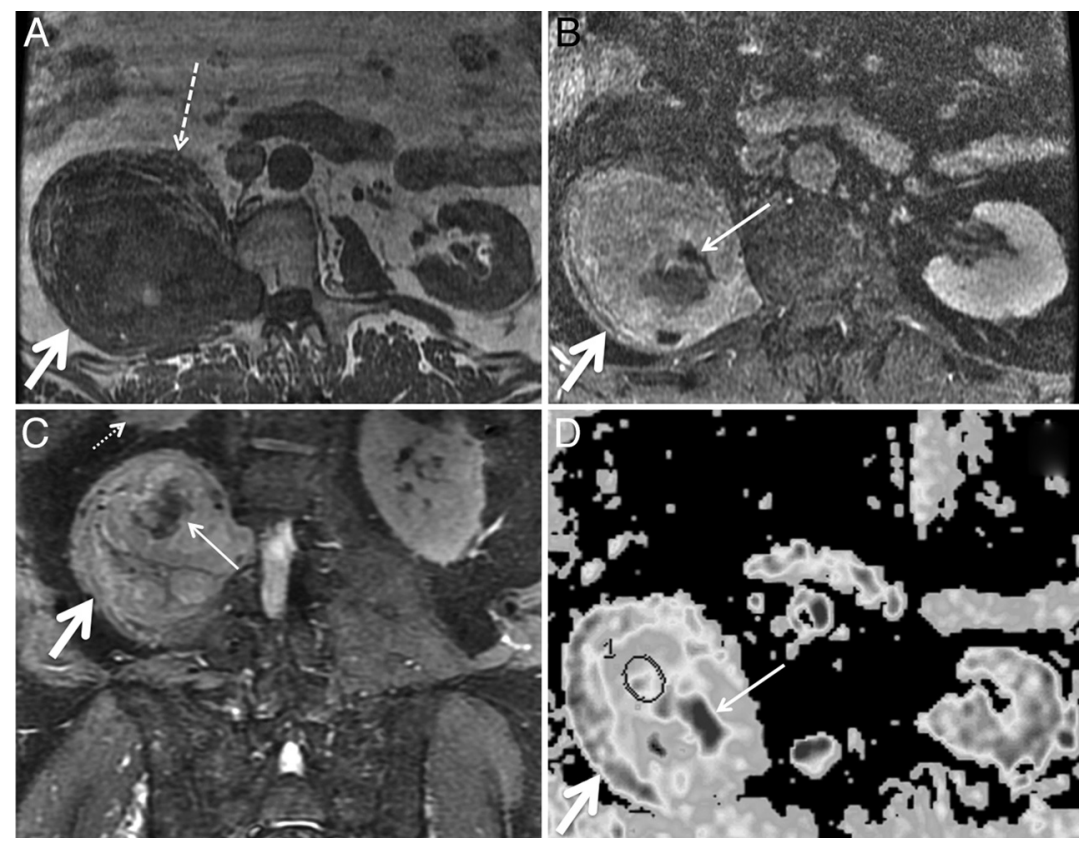

FIG 5. Malignant peripheral nerve sheath tumor in a 69-year-old man with several years of right lower extremity pain and anterior thigh numbness. Percutaneous biopsy several years earlier had demonstrated benign schwannoma. Gross total resection was performed for presumed schwannoma, with areas of necrosis attributed to the large size of the mass and outgrowth of blood supply. Final pathologic diagnosis was malignant peripheral nerve sheath tumor. Axial T1 (A), axial fat-saturated postgadolinium $T 7(B)$, coronal STIR (C), and axial ADC (D) demonstrate a large mass (thick arrows) extending from a widened right $\mathrm{L} 2-\mathrm{L} 3$ neural foramen along the expected course of the right femoral nerve at the posterior aspect of the right psoas muscle. The right psoas muscle (dashed arrow) is draped and elongated over the anterior aspect of the mass. Th right kidney (small dotted arrow) is displaced superiorly. D, ADC ROIs (circle) were selected to avoid nonenhancing areas (thin solid arrows in $B-D$ ) thought to represent necrosis. The mean ADC within nonnecrotic regions in the mass was $0.26 \times 10^{-3} \mathrm{~mm}^{2} / \mathrm{s}$. 

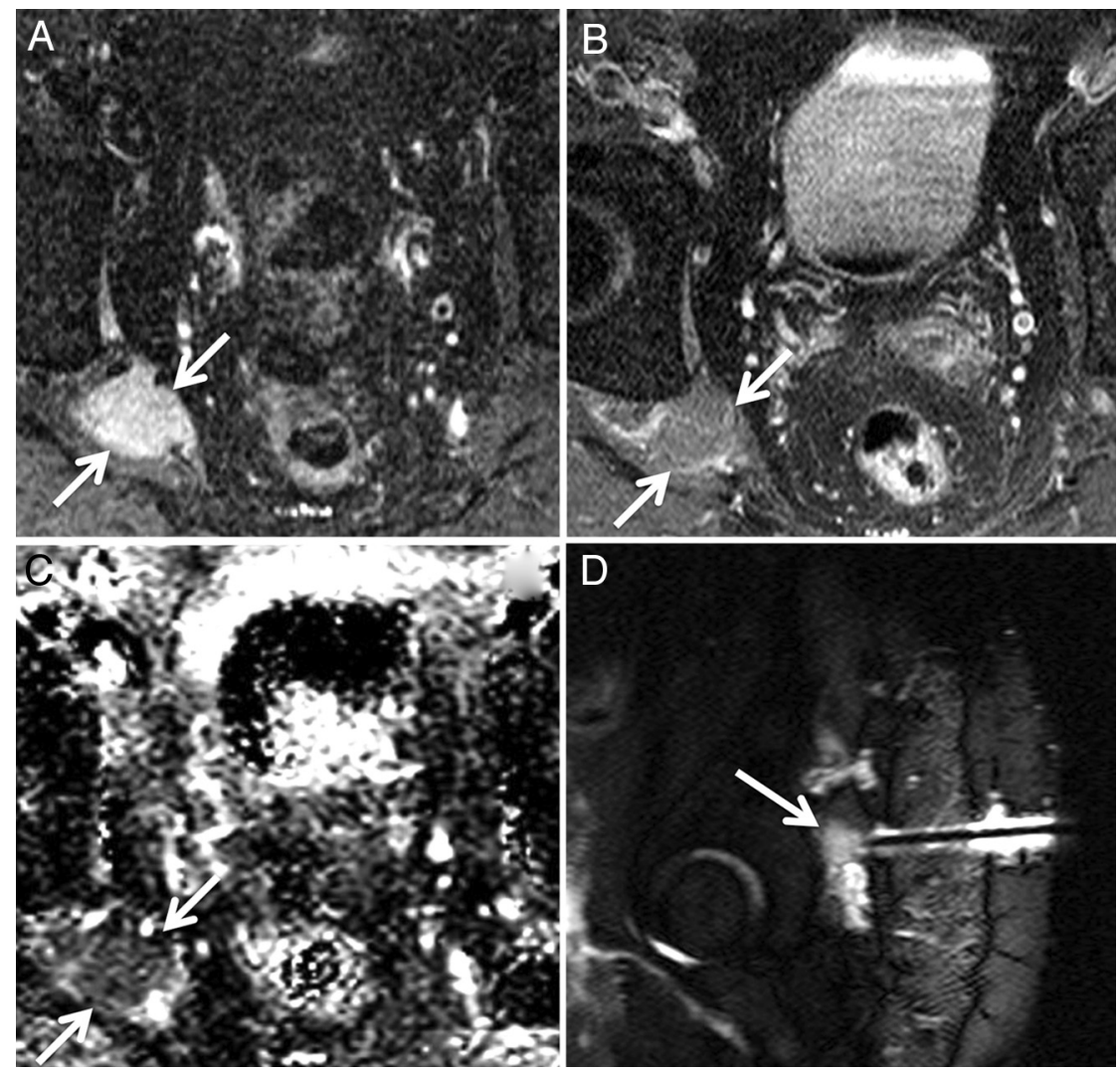

FIG 6. Biopsy-proven non-Hodgkin lymphoma infiltrating the right sciatic nerve. Axial STIR ( $A$ ), axial fat-saturated postgadolinium T1 ( $B)$, and axial ADC map (C) demonstrate a T2 hyperintense minimally enhancing soft-tissue mass (arrows) along the right sciatic nerve at the greater sciatic foramen. ADC within the mass was $0.69 \pm 0.33 \times 10^{-3} \mathrm{~mm}^{2} / \mathrm{s}$. CT-guided biopsy was nondiagnostic. $D, M R$ imaging-guided biopsy was performed, yielding a pathologic diagnosis of diffuse large B-cell lymphoma. The patient underwent chemotherapy and radiation to the mass.
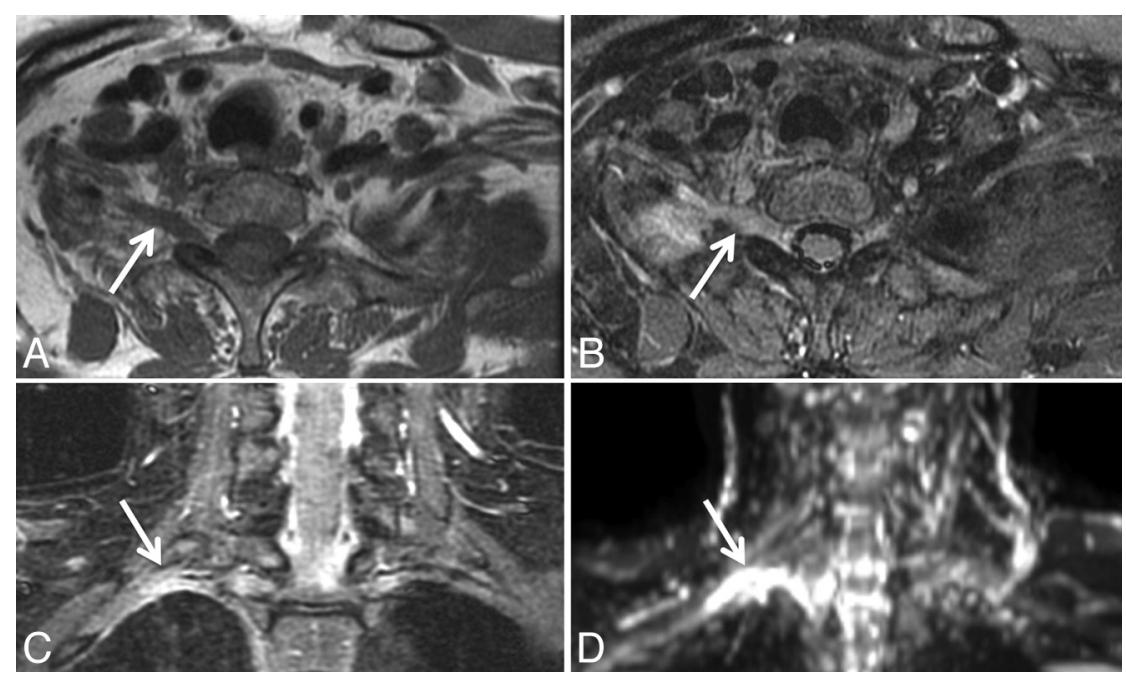

FIG 7. Biopsy-proven metastatic breast cancer infiltrating the right brachial plexus in a 53-year-old woman with right upper extremity pain. Axial $\mathrm{T} T(A)$ and axial STIR (B) images demonstrate a thickened, T2 hyperintense right first thoracic nerve (arrows) with effacement of the fascicular structure normally visible on T1-weighted images. Coronal STIR $(C)$ and maximum-intensity-projection DWI (D) demonstrate asymmetrically increased DWI signal in the right T1 nerve and inferior trunk of the right brachial plexus (arrows), with a corresponding mean ADC of $0.95 \pm$ $0.05 \times 10^{-3} \mathrm{~mm}^{2} / \mathrm{s}$. The patient underwent palliative radiation therapy.

tween separate measurements performed for each lesion by a single radiologist. Interrater reliability was determined through the Pearson correlation coefficient between measurements performed on each lesion by the 2 different radiologists.
One-way between-groups ANOVA was performed to assess for a statistically significant difference in ADC values among the 3 groups (benign lesions, malignant lesions, and postradiation changes) at $P<.05$. Post hoc pair-wise comparisons were 

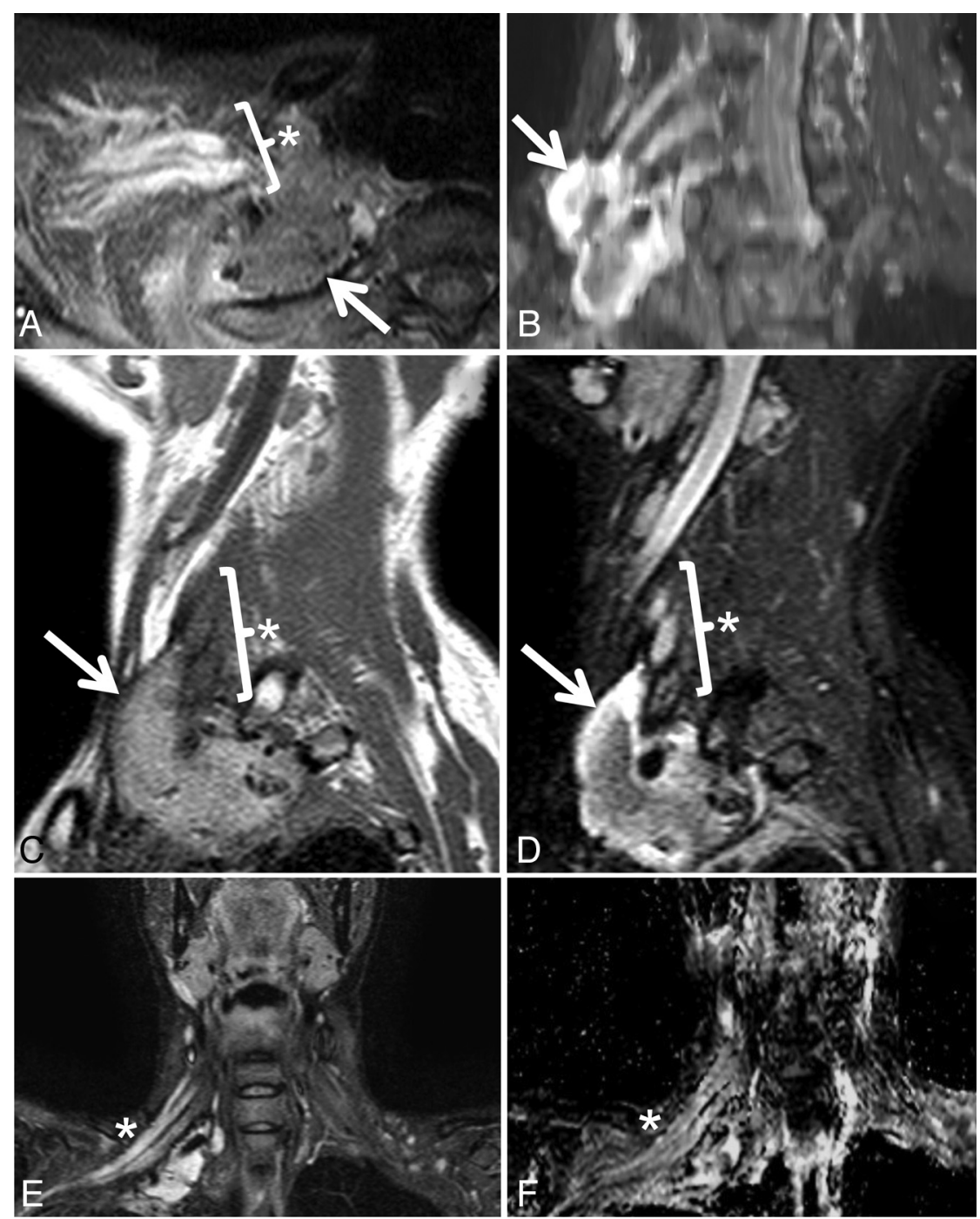

FIG 8. Postradiation brachial plexopathy in a 42-year-old woman with progressive right upper extremity weakness, beginning in the hand and progressing proximally. She had undergone resection and radiation therapy 12 years earlier for high-grade round cell sarcoma of the right brachial plexus. Axial STIR (A), maximum-intensity-projection coronal oblique STIR (B), sagittal TI $(C)$, sagittal STIR $(D)$, coronal STIR $(E)$, and coronal ADC $(F)$ demonstrate a soft-tissue mass (arrows) and a thickened, T2 hyperintense right brachial plexus (asterisks) with an average ADC of $2.59 \pm 0.16 \times 10^{-3} \mathrm{~mm}^{2} / \mathrm{s}$. Although symptoms persisted, the appearance of the mass and the enhancing, T2 hyperintense brachial plexus were unchanged over multiple follow-up MR imaging examinations over 60 months, and a final diagnosis of postradiation brachial plexopathy was made. The soft-tissue mass likely represents fibrosis related to surgical resection of the mass and subsequent radiation.

then performed using the Tukey test to assess for statistically significant pair-wise differences between pairs of groups at $P<.05$.

Although ADC values within each group satisfied the ShapiroWilk test for normality, the sensitivity of this test for nonnormality may be reduced for small sample sizes. ${ }^{17}$ The nonparametric Kruskal-Wallis test was also performed on the ADC values to assess for a statistically significant difference across groups at $P<$ .05. Post hoc nonparametric pair-wise comparisons were performed using Mann-Whitney $U$ tests to assess for statistically significant differences between pairs of groups at $P<.05$. All statistical analyses were performed using SPSS, Version 19 (IBM, Armonk, New York).
This study was approved by the institutional committee on human subjects research. All patient data stored for the purpose of this research study were anonymized by using accession numbers to identify each case.

\section{RESULTS}

The On-line Table shows diagnoses in 23 patients divided into 3 groups on the basis of biopsy results ( $n=10)$; long-term clinical and imaging follow-up $(n=10$; mean follow-up, $40 \pm 31$ months); or intermediate-term follow-up supplemented by PET, neurologic examination, and/or nerve conduction studies $(n=3$, mean follow-up, $4 \pm 1$ months). Group 1 ( $n=$ 10) consisted of benign masses, including 4 biopsy-proven schwannomas, 1 biopsyproven neurofibroma, and 5 peripheral nerve masses that demonstrated both long-term stability in size and conventional MR imaging features characteristic of a benign primary nerve sheath tumor. Group $2(n=7)$, malignant tumors, consisted of 1 biopsy-proven malignant peripheral nerve sheath tumor, 1 biopsyproven rhabdomyosarcoma, 2 cases of metastatic breast cancer involving the brachial plexus (biopsy-proven) or the sacral nerves, 1 case of biopsy-proven metastatic renal cell carcinoma involving a cervical nerve, 1 case of biopsy-proven non-Hodgkin lymphoma involving the sciatic nerve, and 1 case of acute lymphoblastic leukemia involving the brachial plexus. Group $3(n=6)$ consisted of postradiation changes without evidence of residual tumor in 6 cases of previously irradiated malignancies, including 3 cases of breast cancer, 1 highgrade sarcoma, 1 case of Hodgkin lymphoma, and 1 case of oral squamous cell carcinoma. Lesion volumes ranged from 1 to $5530 \mathrm{~cm}^{3}$ (median, $37 \mathrm{~cm}^{3}$; 25th percentile, $14 \mathrm{~cm}^{3}$; 75 th percentile, $\left.122 \mathrm{~cm}^{3}\right)$.

Fig 1 demonstrates ADC values within Groups 1-3. Postradiation changes without evidence of residual tumor (Group 3) demonstrated the highest median ADC value of $2.50 \times 10^{-3} \mathrm{~mm}^{2} / \mathrm{s}$ (interquartile range $=0.87 \times 10^{-3} \mathrm{~mm}^{2} / \mathrm{s}$, minimum $=1.39 \times$ $10^{-3} \mathrm{~mm}^{2} / \mathrm{s}$, maximum $=3.22 \times 10^{-3} \mathrm{~mm}^{2} / \mathrm{s}$, mean ADC $=$ $2.37 \times 10^{-3} \mathrm{~mm}^{2} / \mathrm{s}, \mathrm{SD}=0.61 \times 10^{-3} \mathrm{~mm}^{2} / \mathrm{s}$ ), followed closely by benign lesions (Group 1) with a median ADC value of $1.81 \times$ $10^{-3} \mathrm{~mm}^{2} / \mathrm{s}$ (interquartile range $=0.98 \times 10^{-3} \mathrm{~mm}^{2} / \mathrm{s}$, minimum $=1.30 \times 10^{-3} \mathrm{~mm}^{2} / \mathrm{s}$, maximum $=2.97 \times 10^{-3} \mathrm{~mm}^{2} / \mathrm{s}$, mean $\left.\mathrm{ADC}=1.99 \times 10^{-3} \mathrm{~mm}^{2} / \mathrm{s}, \mathrm{SD}=0.60 \times 10^{-3} \mathrm{~mm}^{2} / \mathrm{s}\right)$. Malignant lesions (Group 2) recorded the lowest median ADC 

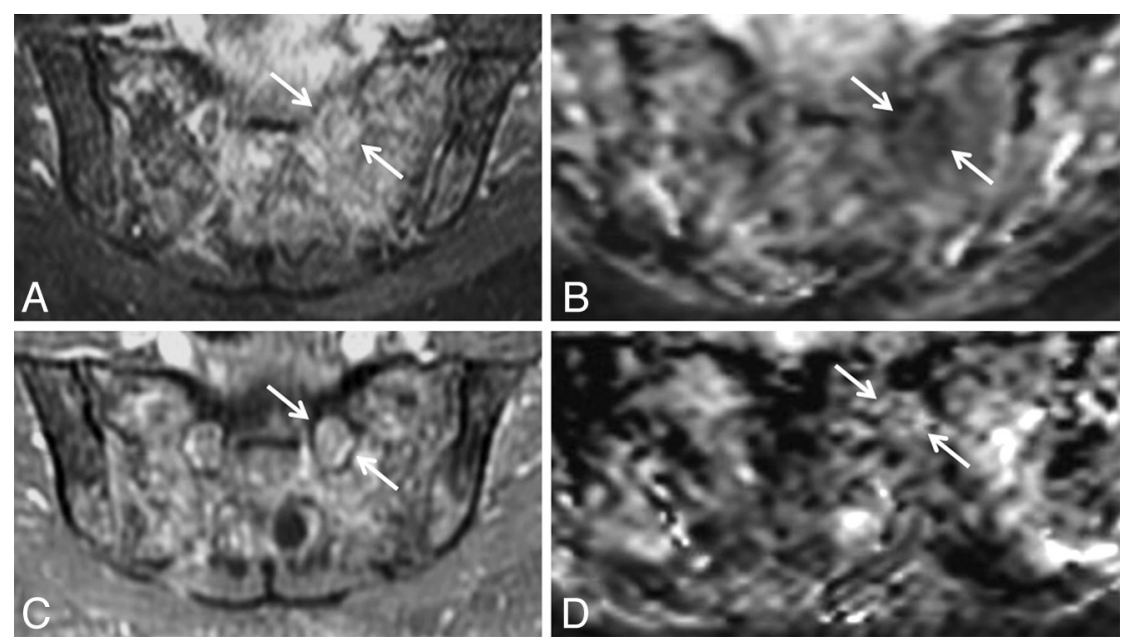

FIG 9. Marked increase in diffusivity following radiation therapy for metastatic breast cancer in a 47-year-old woman with pain in the left L4 through S2 distribution. Axial fat-saturated postgadolinium $\mathrm{Tl}$ and $\mathrm{ADC}$ before $(A$ and $B)$ and 4 months following completion of radiation therapy $(C$ and $D)$. Initial images $(A$ and $B)$ demonstrate metastatic disease throughout the sacrum and infiltrating the left S1 nerve (arrows). $B$, Before radiation, ADC within the diseased left $\mathrm{S} 1$ nerve was $0.78 \pm 0.15 \times 10^{-3} \mathrm{~mm}^{2} / \mathrm{s}$. $D$, Four months later, following completion of radiation therapy, $A D C$ within the nerve had markedly increased to $1.39 \pm 0.23 \times 10^{-3} \mathrm{~mm}^{2} / \mathrm{s}$.

value of $0.69 \times 10^{-3} \mathrm{~mm}^{2} / \mathrm{s}$ (interquartile range $=0.49 \times 10^{-3}$ $\mathrm{mm}^{2} / \mathrm{s}$, minimum $=0.26 \times 10^{-3} \mathrm{~mm}^{2} / \mathrm{s}$, maximum $=1.08 \times$ $10^{-3} \mathrm{~mm}^{2} / \mathrm{s}$, mean ADC $=0.69 \times 10^{-3} \mathrm{~mm}^{2} / \mathrm{s}, \mathrm{SD}=0.28 \times$ $\left.10^{-3} \mathrm{~mm}^{2} / \mathrm{s}\right)$. Intrarater and interrater reliability for ADC measurements, characterized by the Pearson correlation coefficient $r$, were $r=0.98\left(P=7.0 \times 10^{-7}\right)$ for intrarater reliability and $r=$ $0.88(P=.0011)$ for interrater reliability.

One-way between-groups ANOVA demonstrated a statistically significant difference in ADC values across the 3 groups: $F$ $(d f 1=2, d f 2=20)=19.0, P=.000023$. Post hoc pair-wise comparisons using the Tukey test showed that the mean ADC values for Group $1\left(1.99 \times 10^{-3} \mathrm{~mm}^{2} / \mathrm{s}\right)$ and Group $2(0.69 \times$ $\left.10^{-3} \mathrm{~mm}^{2} / \mathrm{s}\right)$ were significantly different at $P=.0002$ and mean ADC values for Group $2\left(0.69 \times 10^{-3} \mathrm{~mm}^{2} / \mathrm{s}\right)$ and Group $3\left(2.37 \times 10^{-3} \mathrm{~mm}^{2} / \mathrm{s}\right)$ were significantly different at $P=.000039$. The ADC for Groups 1 and 3 did not differ significantly from each other $(P=.36)$.

The nonparametric Kruskal-Wallis test also showed a statistically significant difference in ADC across the 3 groups, with $\chi^{2}$ $(d f=2,23$ subjects $)=15.0$ and $P=.00056$. Finally, post hoc nonparametric pair-wise comparisons using the Mann-Whitney $U$ test showed a statistically significant difference in ADC between Groups 1 and $2(P=.00010)$ and between Groups 2 and $3(P=$ $.0012)$, but no statistically significant difference between Groups 1 and $3(P=.97)$.

Figs 2-9 demonstrate MR images of representative cases from each of the 3 groups (benign lesions, malignant lesions, and postradiation changes).

\section{DISCUSSION}

Peripheral polyneuropathies affect multiple peripheral nerves and are generally caused by systemic diseases, most commonly diabetes, and vitamin deficiency related to alcohol use or pernicious anemia, in the United States and Europe. Polyneuropathies are usually diagnosed through clinical history, physical examination, laboratory studies, nerve conduction studies, and needle electromyography. ${ }^{6,18}$ Unlike polyneuropathies, mononeuropathies and plexopathies are most often due to trauma, nerve compression syndromes at characteristic anatomic locations, mass lesions, and postradiation changes; and imaging can play a decisive role in diagnosis. In a study of MR imaging of symptomatic nontraumatic brachial plexopathy, postradiation change was the most common cause, accounting for $31 \%$ of cases, with metastatic breast cancer and primary or metastatic lung cancer accounting for $24 \%$ and $19 \%$ of cases, respectively. The remaining $26 \%$ of cases were caused by a wide variety of benign and malignant tumors. ${ }^{5}$ Unlike many cases of diffuse polyneuropathy, patients with brachial or lumbosacral plexopathy frequently undergo MR imaging due to the localized distribution of symptoms and presumed localized extent of the pathologic findings. ${ }^{19,20}$

We characterized the diffusivity of masslike or infiltrative lesions of the peripheral nerves discovered on MR imaging performed for a clinical indication of peripheral mononeuropathy or brachial or lumbosacral plexopathy. We demonstrated a statistically significant difference among the diffusivities of benign and malignant tumors and postradiation changes. This result is compatible with other studies that have demonstrated diffusivity to be generally inversely correlated with tumor cellularity and tumor grade in several contexts, including adult and pediatric brain tumors, head and neck masses, lymph nodes, retroperitoneal softtissue masses, ${ }^{10-16,21}$ and MR neurography. ${ }^{9}$ Our results are also consistent with a prior study ${ }^{9}$ in which a statistically significant difference in ADC values between benign $\left(1.85 \pm 0.40 \times 10^{-3}\right.$ $\left.\mathrm{mm}^{2} / \mathrm{s}\right)$ and malignant $\left(0.90 \pm 0.25 \times 10^{-3} \mathrm{~mm}^{2} / \mathrm{s}\right)$ peripheral nerve tumors and tumorlike masses was reported $(P<.001)$. In the current study, we extended the analysis to include infiltrative postradiation changes of the peripheral nerves that were not included in that previous study. We report a complete separation of ADC values between benign and malignant lesions, with malignant lesions demonstrating ADC $\leq 1.08 \times 10^{-3} \mathrm{~mm}^{2} / \mathrm{s}$, and benign lesions demonstrating $\mathrm{ADC} \geq 1.30 \times 10^{-3} \mathrm{~mm}^{2} / \mathrm{s}$.

The apparent diffusion coefficient is a measure of the diffusivity, or microscopic mobility, of water protons in tissue. The difference in ADC values of the lesions in our study was likely due to factors such as tumor cellularity, integrity of cell membranes, nuclear-to-cytoplasmic ratio, and the water content of the extracellular matrix. These have been postulated to account for low diffusivity within malignant solid tumors in prior studies. ${ }^{10-16,21} \mathrm{~A}$ study of benign and malignant extracranial soft-tissue tumors in children showed an inverse relationship between ADC measurements and cellular density derived from histologic analysis, but correlation was only moderate $\left(R^{2}=0.54\right)$, and it was postulated 
that additional factors such as extracellular water content also influence the ADC. ${ }^{22}$ Myxoid matrix is known to be abundant in both schwannomas and neurofibromas. Schwannomas, particularly Antoni B tissue within schwannomas, contain a high water content, due to low cellularity and high mucin and low collagen content, ${ }^{23}$ and this likely accounts for their high diffusivity.

Limitations of this study include the lack of biopsy-proven diagnoses for all patients, the likelihood that diffusion characteristics of postradiation changes evolve with time, and the need for validation in a larger study population. Some malignant peripheral nerve sheath tumors, which may arise either de novo or from malignant degeneration of plexiform neurofibromas, have been shown to have a significant myxoid content; one prior study of soft-tissue masses reported that ADC failed to differentiate benign from malignant myxoid soft-tissue tumors, though only one malignant peripheral nerve sheath tumor was included in that study. ${ }^{21} \mathrm{ADC}$ values in postradiation change are likely to be affected by features such as edema, fibrous-inflammatory reaction, and vascular permeability that are temporally dynamic, particularly in the first few months after treatment. Long-term follow-up imaging rather than biopsy was more often performed for lesions with benign features on conventional imaging, introducing a bias, because biopsy was performed on all lesions that ultimately received a malignant diagnosis but on only a subset of lesions receiving a benign diagnosis. Finally, rare entities such as perineuroma ${ }^{24}$ and posttraumatic neuroma appear as mass lesions of the peripheral nerves, but were not included within our study population.

\section{CONCLUSIONS}

We demonstrate a statistically significant difference in diffusivity between biopsy-proven malignant lesions, and lesions with either biopsy-proven benign histology or stable size and benign features on follow-up imaging. Although our results need to be validated in a larger study population, the pattern of diffusivity values within benign and malignant lesions described here may be helpful in selecting patients for percutaneous tissue sampling, debulking versus en bloc total resection, and/or short-term clinical and imaging follow-up.

Disclosures: Nicholas M. Barbaro—UNRELATED: Grants/Grants Pending: National Institute of Neurological Disorders and Stroke, ${ }^{*}$ Elekta.* *Money paid to the institution.

\section{REFERENCES}

1. Spinner RJ. Outcomes for peripheral nerve entrapment syndromes. Clin Neurosurg 2006;53:285-94

2. Britz GW, Haynor DR, Kuntz C, et al. Carpal tunnel syndrome: correlation of magnetic resonance imaging, clinical, electrodiagnostic, and intraoperative findings. Neurosurgery 1995;37:1097-103

3. Guggenberger R, Markovic D, Eppenberger P, et al. Assessment of median nerve with MR neurography by using diffusion-tensor imaging: normative and pathologic diffusion values. Radiology 2012;265:194-203

4. Keen NN, Chin CT, Engstrom JW, et al. Diagnosing ulnar neuropathy at the elbow using magnetic resonance neurography. Skeletal Radiol 2012;41:401-07

5. Wittenberg KH, Adkins MC. MR imaging of nontraumatic brachial plexopathies: frequency and spectrum of findings. Radiographics 2000;20:1023-32

6. Amato AA, Barohn RJ. Peripheral neuropathy. In: Longo DL, Fauci AS, Kasper DL, et al, eds. Harrison's Principles of Internal Medicine. 18th ed. New York: McGraw-Hill; 2011:3448-72

7. Stoll G, Bendszus M, Perez J, et al. Magnetic resonance imaging of the peripheral nerves. J Neurol 2009;256:1043-51

8. Li CS, Huang GS, Wu HD, et al. Differentiation of soft tissue benign and malignant peripheral nerve sheath tumors with magnetic resonance imaging. Clin Imaging 2008;32:121-27

9. Chhabra A, Thakkar RS, Andreisek G, et al. Anatomic MR imaging and functional diffusion tensor imaging of peripheral nerve tumors and tumorlike conditions. AJNR Am J Neuroradiol 2013;34:802-07

10. Nakayama T, Yoshimitsu K, Irie H, et al. Usefulness of the calculated apparent diffusion coefficient value in the differential diagnosis of retroperitoneal masses. J Magn Reson Imaging 2004;20:735-42

11. Thoeny HC, De Keyzer F, King AD. Diffusion-weighted MR imaging in the head and neck. Radiology 2012;263:19-32

12. Abdel Razek AA, Kandeel AY, Soliman N, et al. Role of diffusionweighted echo-planar MR imaging in differentiation of residual or recurrent head and neck tumors and posttreatment changes. $A J N R$ Am J Neuroradiol 2007;28:1146-52

13. Fornasa F, Nesoti MV, Bovo C, et al. Diffusion-weighted magnetic resonance imaging in the characterization of axillary lymph nodes in patients with breast cancer. J Magn Reson Imaging 2012;36: 858-64

14. Koşucu P, Tekinbas C, Erol M, et al. Mediastinal lymph nodes: assessment with diffusion-weighted MR imaging. J Magn Reson Imaging 2009;30:292-97

15. Yamasaki F, Kurisu K, Satoh K, et al. Apparent diffusion coefficient of human brain tumors at MR imaging. Radiology 2005;235:985-91

16. Kan $P$, Liu JK, Hedlund G, et al. The role of diffusion-weighted magnetic resonance imaging in pediatric brain tumors. Childs Nerv Syst 2006;22:1435-39

17. Shapiro SS, Wilk MB, Chen HJ. A comparative study of various tests for normality. J Am Statist Assoc 1968;63:1343-72

18. England JD, Asbury AK. Peripheral neuropathy. Lancet 2004;363: 2151-61

19. Grant GA, Britz GW, Goodkin R, et al. The utility of magnetic resonance imaging in evaluating peripheral nerve disorders. Muscle Nerve 2002;25:314-31

20. Thyagarajan D, Cascino T, Harms G. Magnetic resonance imaging in brachial plexopathy of cancer. Neurology 1995;45:421-27

21. Nagata S, Nishimura H, Uchida M, et al. Diffusion-weighted imaging of soft tissue tumors: usefulness of the apparent diffusion coefficient for differential diagnosis. Radiat Med 2008;26:287-95

22. Humphries PD, Sebire NJ, Siegel MJ, et al. Tumors in pediatric patients at diffusion-weighted MR imaging. Radiology 2007;245: $848-54$

23. Wippold FJ, Lubner M, Perrin RJ, et al. Neuropathology for the neuroradiologist: Antoni A and Antoni B tissue patterns. AJNR Am J Neuroradiol 2007;28:1633-38

24. Merlini L, Viallon M, De Coulon G, et al. MRI neurography and diffusion tensor imaging of a sciatic perineuroma in a child. Pediatr Radiol 2008;38:1009-12 